\title{
On the Utility of Extended Fingerprint Features: A Study on Pores
}

\author{
Qijun Zhao and Anil K. Jain \\ Department of Computer Science and Engineering \\ Michigan State University \\ East Lansing, MI 48824, U.S.A. \\ \{qjzhao, jain\}@cse.msu.edu
}

\begin{abstract}
Extended fingerprint features are routinely used by latent examiners in forensic applications. They are now being considered for inclusion in automatic fingerprint identification systems (AFIS), particularly with the adoption of 1000ppi resolution in the Next Generation Identification (NGI) system. Earlier studies on this topic suffered from two limitations: (i) experiments were based on live scan images that are generally of good quality and contain smaller intra-class variations compared to ink images and (ii) the baseline minutiae matcher used to measure the additive value of extended features was not a state-of-the-art matcher. In this paper, we study the utility of pores, one of the most prevalent extended fingerprint features, on rolled ink fingerprint images at both 500ppi and 1000ppi resolution in the NIST SD30 database. The results show that the fingerprint image quality significantly affects the automatic extraction and matching accuracy of pores. Furthermore, the contribution of pores to the overall fingerprint recognition accuracy is miniscule when a COTS matcher is used for 500ppi rolled ink fingerprint images. The fusion between pore matcher and COTS minutiae matcher is a bit more effective on 1000ppi good quality rolled ink fingerprint images. We believe that these results will be useful in the design of next generation AFIS.
\end{abstract}

\section{Introduction}

Fingerprint recognition has been accepted as a reliable personal identification technique for almost 100 years. Fingerprints are now routinely used to identify suspects and victims in law enforcement and forensics [1]. The demand for automatic fingerprint identification systems (AFIS) became compelling in the early 1960s, because of the rapid expansion of fingerprint recognition in forensics and the ever-increasing size of fingerprint databases (e.g. the FBI fingerprint database now has more than 800 million fingerprint images) [2]. Many automatic algorithms have been proposed for extracting and matching fingerprint features, and a large number of AFIS

The authors would like to thank Dr. Jianjiang Feng for suggestions during this research. are successfully deployed not only for forensic applications, but also for civilian and commercial applications [1,2]. (a)

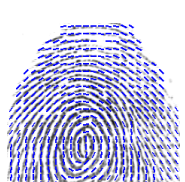

(c)

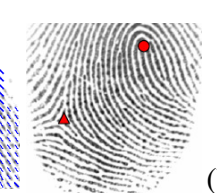

(b)

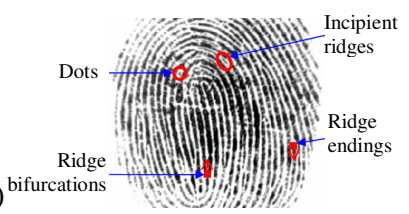

Pores (open)
Fig. 1: Example fingerprint features: (a) level 1 (ridge orientation field and singular points), (b) level 2 (minutiae, dots, and incipient ridges), (c) and level 3 (pores and ridge edge features).

Fingerprint features can be divided into three levels [2], as shown in Fig. 1. Level 1 features are defined by fingerprint ridge flow and general morphological information, e.g. ridge orientation field, ridge pattern types, and singular points. These features are not very unique to each finger and are thus mostly used for fingerprint type classification and indexing. Level 2 features refer to individual fingerprint ridges and fingerprint ridge events, including minutiae, dots, incipient ridges, etc. There are two prominent types of minutiae, i.e. ridge endings and ridge bifurcations. Level 2 features are generally believed to be discriminative, stable, and robust. As a result, commercial fingerprint systems are primarily based on the minutiae features. Level 3 features are defined as fingerprint ridge dimensional features. Pores and ridge edge shapes are typical level 3 features. These features, if reliably extracted, are also quite distinctive [3, 4]. Yet, to our knowledge, they have not been incorporated in commercial systems. The current AFIS technology mainly utilizes the features of the first two levels [2]. Extended fingerprint features are loosely defined as any feature that is not currently used in commercial AFIS.

SWGFAST, the Scientific Working Group on Friction ridge Analysis, Study and Technology, stated that the AFIS technology is utilizing only a limited amount of available fingerprint detail [5]. For example, in the FBI standard minutiae template, the Type 9 minutiae record 
includes only ridge endings and bifurcations [6]. On the contrary, latent fingerprint experts usually rely on additional information. In particular, they routinely explore a variety of extended features in manual latent identification [5]. To improve the accuracy of AFIS and to narrow the performance gap between AFIS and latent experts, SWGFAST suggested exploiting as many fingerprint features as possible. CDEFFS, the Committee to Define an Extended Fingerprint Feature Set, was chartered to define the next ANSI/NIST-ITL standard [7] so that additional features can be utilized in the next generation AFIS. In its report, CDEFFS [7] defined the extended fingerprint feature set, including dots, incipient ridges, pores, creases, and ridge edge features (protrusions and indentations). These features provide additional information to individualize a fingerprint and are claimed to be routinely used by latent experts. Pores, which are abundant on fingerprints, are one of the most prevalent extended features. According to our statistics on NIST SD30, there are, on an average, between 50 to 100 detectable pores in a small area of about $8 \mathrm{~mm} \times 6 \mathrm{~mm}$ on 500ppi and 1000ppi good quality fingerprint images.

A number of researchers have investigated the use of pores in AFIS by using live-scan fingerprint images. Stosz and Alyea proposed the first pore-based fingerprint matcher [8]. Kryszczuk et al. studied the effectiveness of pores in matching small fragmentary fingerprints with full fingerprint templates [9]. Jain et al. utilized fingerprint features at all the three levels, including minutiae and pores [10]. Zhao et al. proposed pore extraction and matching methods and applied them to partial and full high resolution fingerprint matching [11, 12]. All these studies reported that fusion of pores with minutiae improves the fingerprint matching accuracy. However, a common characteristic of these studies is that they all used live-scan fingerprint images with resolution of at least 1000ppi. As a result, the quality of the fingerprint images used in these studies is generally very good making it easy to extract pores. In addition, these images were collected using the same fingerprint sensor and the capture interval between different samples is small, resulting in small intra-class variations. The same conditions may not hold true for ink fingerprint images that are included in almost all the forensics and law enforcement databases. Another drawback of these studies is that the usefulness of pores was shown by comparing pore matching accuracy to an inhouse minutiae matcher instead of a commercial off the shelf (COTS) matcher.

Fingerprint images can be broadly classified into three categories: live-scan, ink, and latent (see Fig. 2). Live-scan fingerprint images are obtained by using optical, capacitive or other types of sensors to directly image the finger. Usually, live-scan images are captured under controlled conditions and are easy to recapture, and as a result, their quality, on average, is the best among the (a)

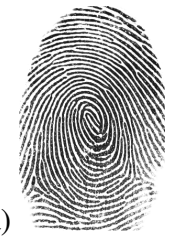

(c)

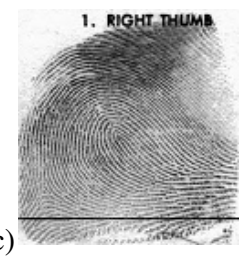

(e)

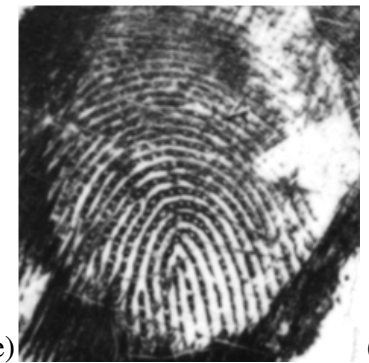

(d)
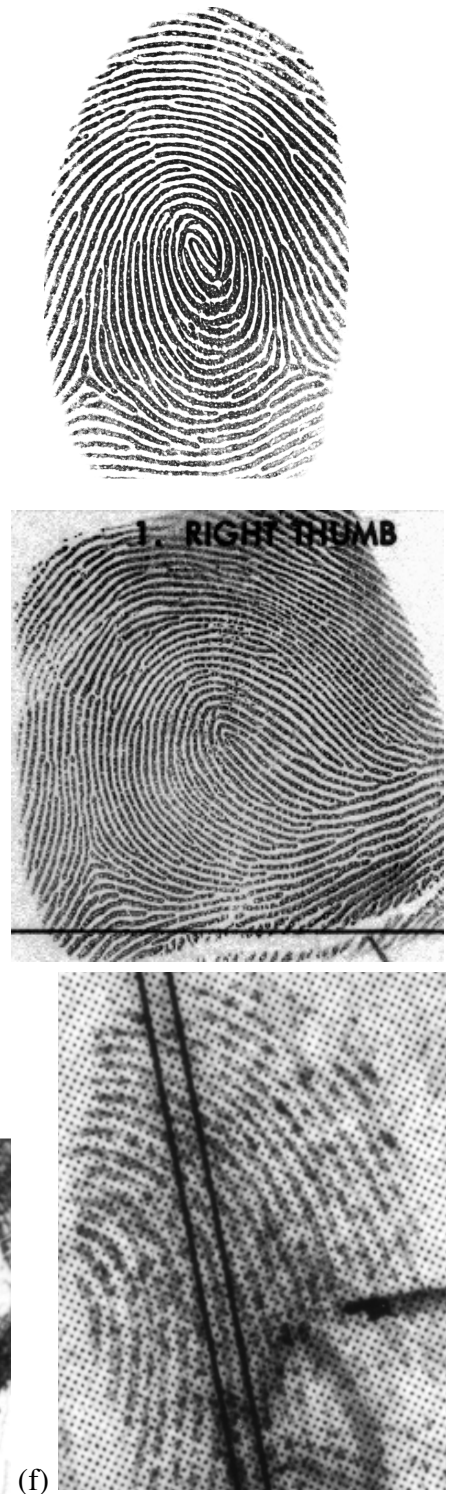

Fig. 2: Three types of fingerprints. (a) and (b) 500ppi and 1000ppi live-scan fingerprint images collected by CrossMatch $1000 \mathrm{ID}$ scanner. (c) and (d) 500ppi and 1000ppi ink fingerprint images in NIST SD30. (e) and (f) two 1000ppi latent fingerprint images in NIST SD27.

three kinds of fingerprint images. Given a sufficiently high resolution image (e.g. 1000ppi), the pores in live-scan fingerprint images are very clear and can be reliably extracted (see Figs. 2(a) and 2(b)). Rolled Ink fingerprint images are widely used in forensics. To capture the ink fingerprint images of a subject, his/her finger is coated with black ink and pressed or rolled against a paper card. The print left on the paper card is then scanned to digital format. In general, the quality of ink fingerprint images is lower than live-scan fingerprint images. The pores in ink fingerprint images are often not very clear, making it difficult to reliably locate the pores (see Figs. 2(c) and 2(d)). Latent fingerprint images are inadvertently left by persons on surfaces of objects and are lifted or 
photographed by using various techniques, e.g. chemical processing [1]. Latent fingerprint images are characterized by small area, poor quality, and large non-linear distortion [13]. Consequently, the extended features like pores can be quite vague and unreliable as shown in Figs. 2(e) and 2(f). This was also observed in [13], which reported no obvious improvement in the latent matching accuracy by using pores in the NIST SD27 images. Based on the ground truth of extended features (i.e. pores, dots, and incipient ridges) marked on the fingerprint images in NIST SD27 by a latent expert, Jain and Feng [13], reported that the number of mated pores is much larger than dots and incipient ridges (there can be more than 20 mated pores between a latent and the corresponding mated rolled print, but only about 5 mated dots and incipient ridges). Moreover, some fingers do not even have any dots or incipients, whereas all fingers have pores on them. We therefore mainly focus on the discriminative ability of pores in this paper.

The results of previous studies have shown that the performance of pores can be significantly affected by the fingerprint image quality. We believe that currently, there is no consensus on the utility of fingerprint pores when a large variation in fingerprint image quality exists across the database. Note that in [10], the authors applied their pore-based matcher to low and high quality fingerprint images separately and reported improvement in both the cases. But because they used live-scan images, even their "low" quality images were, on average, still better than the "best" ink fingerprint images in NIST SD30 considered here.

The goal of this paper is to study the utility of fingerprint pores in the context of varying fingerprint image quality in operational databases. In particular, we will use the dual resolution (500ppi and 1000ppi) rolled ink fingerprint images in NIST SD30 for our experiments. The main contributions of this paper are:

- Design and compare automatic pore extraction using different quality fingerprint images. We show that low quality fingerprint images lead to many false detections, while the true detection accuracy does not significantly change across different quality images;

- Study the fusion of pore matcher and minutiae matcher. A commercial minutiae matcher (Verifinger by Neurotechnology [16]), instead of an in-house minutiae matcher, is used in our study. The results show that the contribution of pores to the accuracy improvement of minutiae matcher is minor for 500ppi fingerprint images, but more noticeable for 1000ppi good quality fingerprint images.

- Study the impact of resolution (500 ppi vs. 1000ppi) on fingerprint recognition accuracy. It is shown that while the increased resolution improves the accuracy of both matchers, the improvement to minutiae matcher is more significant than pore matcher. The fusion of pore and minutiae matchers is also more effective for high resolution (1000ppi) fingerprint images, but only when the fingerprint image quality is good.

The rest of this paper is organized as follows. Section 2 introduces automatic pore extraction and matching algorithms. Section 3 introduces the experimental design, including the database, image quality measure, and the evaluation methods. Section 4 then presents and analyzes the experimental results. Section 5 concludes the paper.

\section{Pore extraction and matching}

Pores, also known as sweat pores, are located on finger ridges. They are formed in the sixth month of gestation due to the sweat-gland ducts reaching the surface of the epidermis. Once the pores are formed, they are fixed on the ridges and there can be between 9 to 18 pores along a centimeter of ridge [2]. A pore can be visualized as open on one print, but as closed on the other print depending on pressure and whether it is exuding perspiration. As shown in Fig.1(c), a closed pore appears as an isolated dot on the ridge, while an open pore is connected to one or both of the two valleys surrounding it. As a result, the shape and size of a pore can vary from one impression to another, and therefore only its position is used in pore matching. In this section, we will introduce automatic algorithms for pore extraction and matching.

\subsection{Pore extraction}

It can be seen from Fig. 1(c) that closed pores are isotropic, whereas open pores are anisotropic. Compared to other methods in the literature which are based on isotropic models, the proposed anisotropic pore model [11] can better cope with the open or closed status of pores and has a better pore detection performance. The rationale of this model is that along the ridge orientation at a pore, the intensity profile across the pore has a Gaussian shape irrespective of whether it is open or closed. The anisotropic pore model is defined as:

$$
\begin{gathered}
\left\{\begin{array}{c}
P_{0}(i, j)=e^{-\frac{j^{2}}{2 \sigma^{2}}} \cdot \cos \left(\frac{\pi}{3 \sigma} i\right) \\
-3 \sigma \leq i, j \leq 3 \sigma
\end{array}\right. \\
\left\{\begin{array}{c}
P_{\theta}(i, j)=\operatorname{Rot}\left(P_{0}, \theta\right)=e^{-\frac{\hat{j}^{2}}{2 \sigma^{2}}} \cdot \cos \left(\frac{\pi}{3 \sigma} \hat{i}\right) \\
\hat{i}=i \cos (\theta)-j \sin (\theta), \hat{j}=i \sin (\theta)+j \cos (\theta) \\
-3 \sigma \leq i, j \leq 3 \sigma
\end{array}\right.
\end{gathered}
$$

Here, $P_{0}$ models the pores located on horizontal ridges, and $P_{\theta}$ the pores on ridges of orientation $\theta$. The two parameters, orientation $\theta$ and scale $\sigma$, can be determined by the local ridge orientation and the local ridge period, respectively. To apply the pore model, the image is first divided into non-overlapping blocks. For each foreground 
block that has dominant ridge orientation, a local instantiation of the model is established based on the local ridge orientation and frequency in the block, and then convolved with the block as a pore matched filter. An empirically chosen threshold is then applied to the filter response, resulting in a binary image in which pixels corresponding to candidate pores have value 1 , whereas the other pixels have value 0 . Post-processing steps are applied to remove possible false detections, including removing pores which are not on ridges, pores which have very low intensity, and pores whose size are not in a prespecified range. We refer the readers to [11] for more detail of the method. Figs. 3(a) and 3(b) show the extracted pores in a pair of mated fingerprint images in NIST SD30 (cropped for display purpose).

\subsection{Pore matching}

The extracted pores are matched using the method in [12]. This method has the advantage that the pore matching is independent of minutiae matching which enables more effective fusion between the two match scores [12]. Given two sets of pores, the individual pores are compared first based on the correlation between their local neighborhoods in the corresponding images. In our experiments, neighborhoods of radius 15 and 25 pixels are used for 500ppi and 1000ppi images, respectively. The coarse correspondences between pores are then refined to remove possible false correspondences by using the RANSAC (RANdom SAmple Consensus) algorithm [12] under the assumption of affine transformation between the two fingerprints. The number of the final pore correspondences is defined as the pore match score between the two fingerprints. Fig. 3(c) shows the pore correspondences in Figs. 3(a) and 3(b).

\section{Experimental design}

\subsection{Dataset}

There is no public domain fingerprint database available with ground truth for extended features. So, we created our own ground truth data to evaluate the pore extraction algorithm. Manually marking extended features in fingerprint images is both time consuming and tedious which explains our small sized evaluation database of partial fingerprints. The NIST SD30, used in this study, contains 720 rolled ink fingerprint images of 360 fingers, captured at two different sessions at both 500ppi and 1000ppi resolution. Example fingerprint images from this database are shown in Figs. 2(c) and 2(d). We created 30 partial fingerprint images (320 by 240 pixels) by cropping the 1000ppi fingerprint images in NIST SD30 which display various quality values (see the next section). The pores on these partial fingerprint images were then manually marked as ground truth for the evaluation of automatic pore detector.

(a)

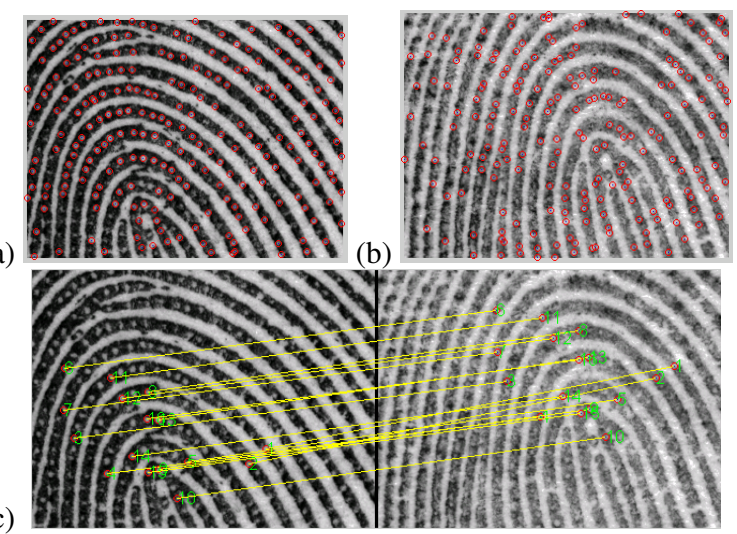

Fig.3: Pore extraction and matching. (a) and (b) show a pair of mated fingerprint images with extracted pores marked by circles, and (c) the corresponding pores in (a) and (b).

\subsection{Fingerprint image quality}

In order to investigate the utility of pores with varying quality of fingerprint images, we divided the fingerprint images into three quality groups: good, medium and bad. The NFIQ measure [14] is widely used in the literature, but it was initially designed for plain fingerprint images. Therefore, in this study, we used the symmetric derivative based fingerprint image quality assessment method proposed in [15]. By using the symmetric derivatives, parallel ridges/valleys on fingerprints can be modeled as linear symmetric features, while singular points (i.e. cores and deltas) are parabolic symmetric features. For good quality fingerprint images, a local patch is usually characterized by either linear symmetry or parabolic symmetry. In contrast, noisy or low-contrast regions will display both symmetries. Based on this property, a fingerprint image is first divided into blocks, two filters are used to detect the two kinds of symmetries in each block, and the quality of each block is defined by the correlation between the responses of the two filters. The quality of a fingerprint image is defined as the average of the quality values of all its blocks.

Given a quality measure, the fingerprint images in the database were divided into three groups (good, medium and bad). From each group, 10 fingerprint images were randomly chosen to construct the ground truth dataset of the corresponding quality level. Fig. 4 shows three example fingerprint image fragments which belong to good, medium, and bad quality ground truth dataset, respectively. The ground truth pores and the detected pores on them are marked by white dots and red circles.

In fingerprint matching, all the 360 fingerprint images from the first session were matched with all the 360 fingerprint images in the second session, resulting in 360 genuine pairs and 129,240 imposter pairs. The quality of 
each pair was defined as the minimum of the quality of the two fingerprint images in the pair. The genuine and imposter pairs were sorted according to their decreasing quality value. These were then divided into three equal sized groups of good, medium and bad (120 genuine pairs and 43,080 imposter pairs in each group).

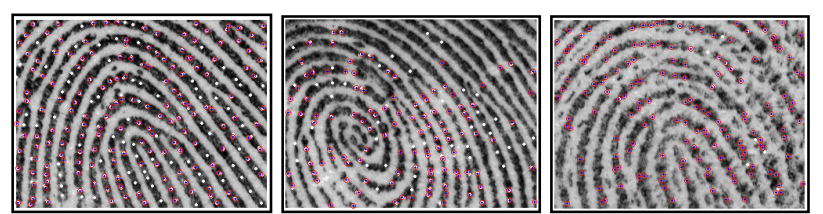

Fig. 4: Examples of good, medium, and bad quality fingerprint images (from left to right) in the ground truth datasets. White dots and red circles denote the ground truth and detected pores.

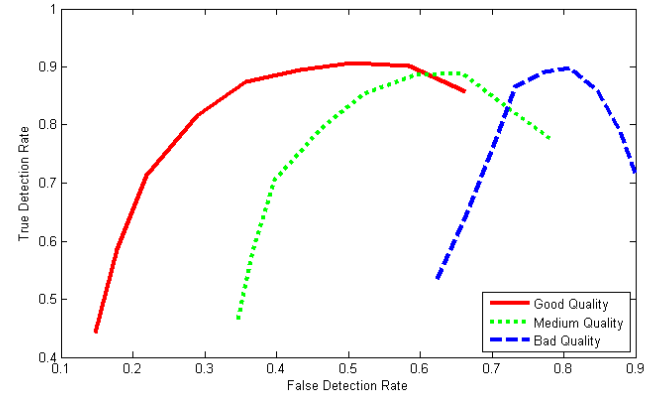

Fig. 5: Pore detection accuracy on different quality groups when different thresholds were applied to the pore matched filter responses.

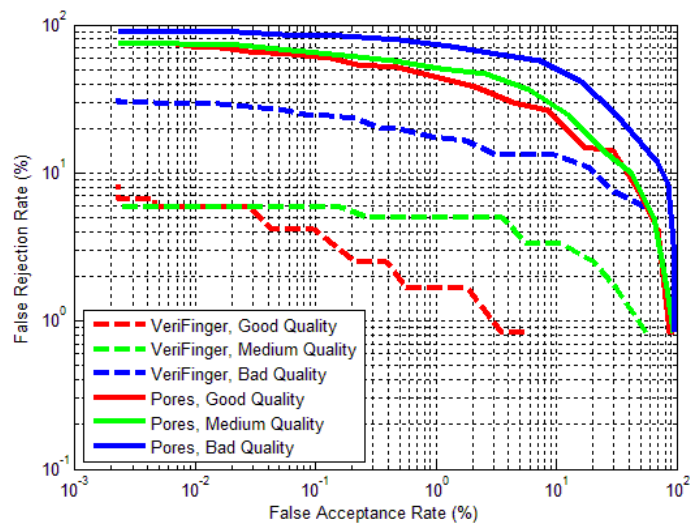

Fig. 6: The DET curves of pore matcher and VeriFinger on 500ppi rolled ink fingerprint images.

\subsection{Evaluation methods}

The automatic pore detection accuracy is evaluated by using the true detection rate $\left(R_{t}\right)$ and false detection rate $\left(R_{f}\right) . R_{t}$ is defined as the ratio of the number of true detected pores to the total number of ground truth pores and $R_{f}$ is defined as the ratio of the number of falsely detected pores to the total number of detected pores. In our experiments, given a detected pore, if there is a true pore within a distance of five pixels, it is considered a true detected pore. The receiver operating characteristic (ROC) curves that plot $R_{t}$ vs. $R_{f}$ when different thresholds were applied to the pore matched filter responses are calculated for all the three quality groups.

For evaluating matching accuracy, the detection error trade-off (DET) curve that plots the false rejection rates (FRR) at a few specific false acceptance rates (FAR) is used. In order to investigate the performance of combining pores with minutiae, a commercial minutiae-based fingerprint matcher, VeriFinger [16], is used. The minutiae and pore match scores are combined using the weighted sum rule that has been reported in the literature to give good results especially when a small number of match scores are available [17]. We did not use more complicated fusion methods such as the likelihood ratio based method [18], which need a large number of training samples.

\section{Results and analysis}

\subsection{Pore detection accuracy}

Fig. 5 shows the ROC (i.e. $R_{t}$ vs. $R_{f}$ ) curves of the pore detection accuracy on the three quality groups. While the best true detection rate does not change significantly across different quality groups, the false detection rate, on average, increases as the fingerprint image quality decreases. Given a true detection rate of $70 \%$, the false detection rate is $\sim 20 \%, \sim 40 \%$, and $\sim 70 \%$ for the good, medium, and bad quality groups, respectively. As can be seen in Fig. 4, noise and degradation of ridge/valley structures in poor fingerprint images lead to detection of many spurious pores.

\subsection{Pore matching accuracy}

The DET curves of pore matching alone on the three different quality groups of 500ppi rolled ink fingerprint images are plotted in Fig. 6. For comparison, the DET curves of VeriFinger minutiae matcher are also shown. Note that the recognition accuracy of both pore matcher and VeriFinger decreases as the fingerprint image quality degrades. The accuracy of pore matcher is significantly worse than that of VeriFinger on all the three quality groups. While for good quality fingerprint images, pore matching accuracy is much worse than VeriFinger, for bad quality images, the performance difference between pore matcher and VeriFinger becomes small. This vast difference between the accuracy of pore matcher and VeriFinger also affects the fusion of pores and minutiae as shown in the next section.

\subsection{Combining minutiae and pores}

The weighted sum fusion method [17] was employed to combine the match scores of pores and VeriFinger. The 
DET curves before and after fusion are shown in Fig. 7. From these results, we can see that when the fingerprint image quality is bad, the fusion of pores with VeriFinger minutiae matcher leads to some improvement in the recognition accuracy; on the other hand, if the fingerprint image quality is good, including the pores either does not improve the recognition accuracy, or even degrades the recognition.

(a)
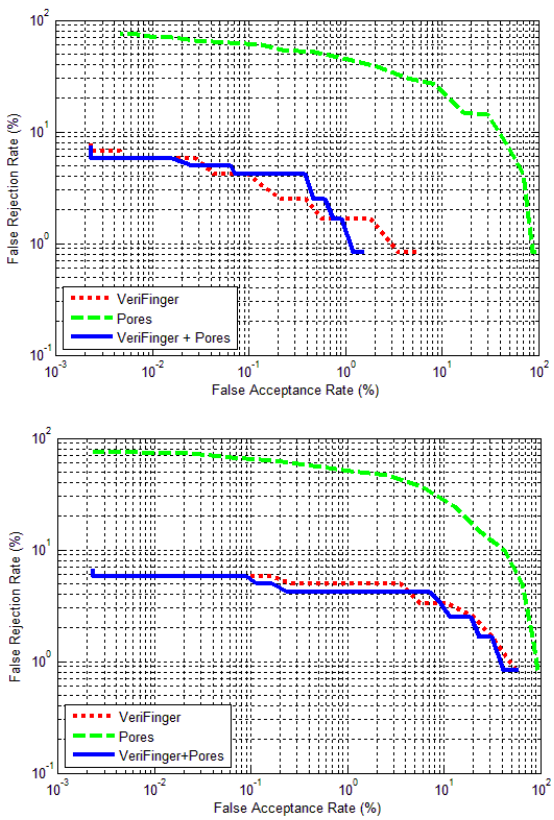

(b)

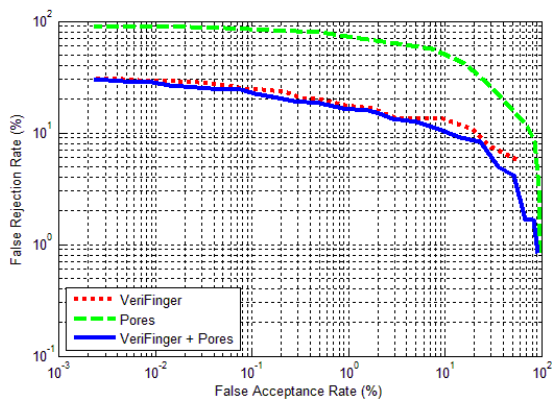

(c)

Fig. 7: The DET curves before and after fusion by weighted sum fusion method on 500ppi rolled ink fingerprint images of (a) good, (b) medium, and (c) bad quality.

Given that the accuracy of pore matcher on the 500ppi fingerprint images is quite poor compared to minutiae matcher (at FAR of $0.01 \%$, the FRRs of VeriFinger are $5.83 \%, 5.83 \%$, and $29.17 \%$ on good, medium, and bad quality groups, respectively, but $71.67 \%, 76.67 \%$, and $89.17 \%$ for pores), the fusion results are not surprising; it is well known that a weak classifier can degrade the accuracy of a very strong classifier. The FRRs after fusion of VeriFinger and pores become 5.83\%, 5.83\%, and $28.33 \%$ on the three quality groups. The improvement made by pores to the recognition accuracy, if any, is minute.

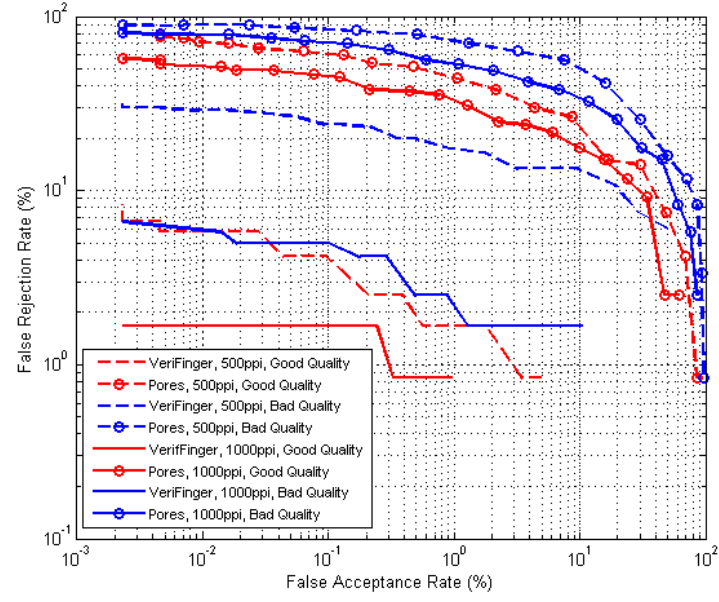

Fig. 8: The DET curves of VeriFinger and Pores on 1000ppi and 500ppi fingerprint images in different quality groups.

\subsection{Impact of resolution}

The above recognition experiments used 500ppi rolled ink fingerprint images. In the literature, it has been claimed that at least $800 \mathrm{ppi}$ is required to accurately detect pores [8] and almost all the existing studies employed live-scan fingerprint images of $1000 \mathrm{ppi}$ or greater resolution. In order to evaluate the impact of fingerprint image resolution on the utility of pores, we also repeated the above recognition experiments by using the 1000ppi rolled ink fingerprint images in NIST SD30. The DET curves of pores and VeriFinger on the good and bad quality groups are shown in Fig. 8 (for comparison, the results on 500ppi fingerprint images are also given). These curves demonstrate that the recognition accuracy of the minutiae-based matcher is significantly improved when the fingerprint image resolution is increased from 500ppi to $1000 \mathrm{ppi}$, whereas the improvement in the accuracy of the pore-based matcher is relatively small. The average improvement over FMR100, FMR1000 and FMR10000 (i.e. $\mathrm{FRR}$ at $\mathrm{FAR}=1 \%, \mathrm{FAR}=0.1 \%$ and $\mathrm{FAR}=0.01 \%$ ) on the good, medium and bad quality groups is $(27.52 \%$, $28.48 \%, 18.35 \%)$ and $(60.54 \%, 75.37 \%, 80.72 \%)$ for pores and VeriFinger, respectively. It is interesting that the pore-based matcher achieves bigger improvement on good quality fingerprint images than bad quality fingerprint images, and on the contrary, the minutiae-based matcher obtains larger improvement on bad quality fingerprint images. This might be because for bad quality fingerprint images, increasing the image resolution would make very little contribution to improving the usability of pores, which are fine details on fingerprint ridges. On the other hand, the ridge and valley structures on bad quality fingerprint images can be effectively improved by higher image resolution. Because minutiae are defined by ridge path events, the accuracy of the minutiae-based matcher on bad quality fingerprint images is thus greatly improved 
when higher resolution fingerprint images are used.

(a)
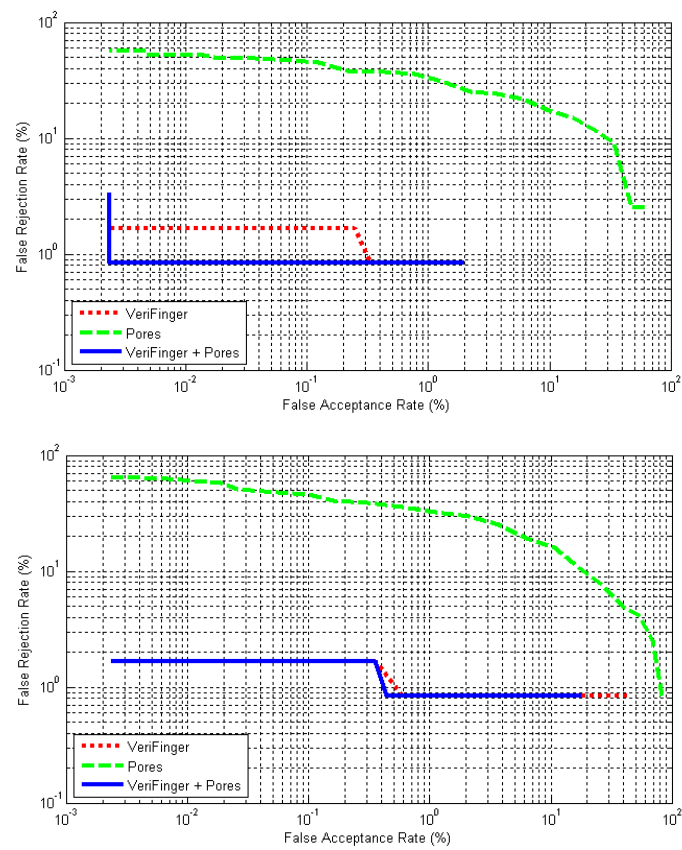

(b)

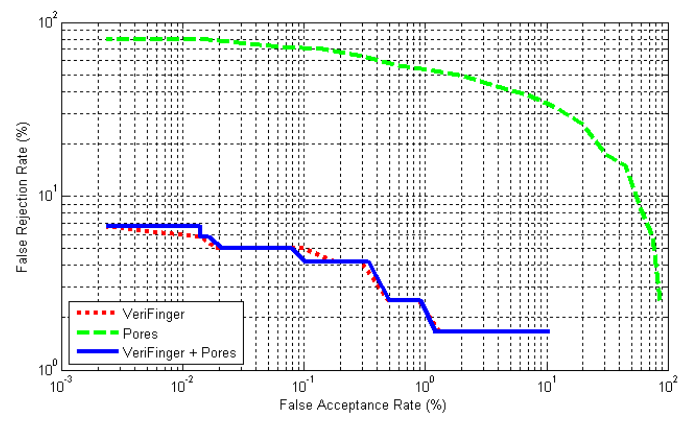

(c)

Fig. 9: The DET curves before and after fusion by weighted sum fusion method on 1000ppi rolled ink fingerprint images of (a) good, (b) medium, and (c) bad quality.

The pore-based and minutiae-based match scores of the 1000ppi rolled ink fingerprint images were fused by using the weighted sum fusion method [17]. The corresponding DET curves are presented in Fig. 9 for the good, medium, and bad quality groups. Comparing these curves with those in Fig. 7, we can see that the fusion results differ on 500ppi and 1000ppi rolled ink fingerprint images. While the fusion between pores and minutiae does not make any noticeable improvement on 500ppi fingerprint images, the fusion is more effective on 1000ppi good quality fingerprint images. We believe that this is due to the different contributions of increased resolution to the accuracy of the pore-based and minutiae-based matchers on different quality fingerprint images. Moreover, when 500ppi fingerprint images are used, the fusion makes very minor improvement in the recognition accuracy of VeriFinger, i.e. about $2.88 \%$ for FMR10000 (decreasing from $29.17 \%$ to $28.33 \%$ ) on bad quality fingerprint images and no improvement on medium and good quality fingerprint images. In contrast, the improvement can be up to about $50 \%$ (decreasing from $1.67 \%$ to $0.83 \%$ ) on 1000 ppi good quality fingerprint images. These results demonstrate that the utility of pores is related to the fingerprint image quality and can be improved by using high resolution fingerprint images.

Fig. 10 gives an example of successfully using pores to improve the recognition, where one genuine pair and one imposter pair of $1000 \mathrm{ppi}$ good quality rolled ink fingerprint images in NIST SD30 are shown. As can be seen in Fig. 10(a) and Fig. 10(c), VeriFinger generated a higher match score for the imposter pair (0.0249) than for the genuine pair (0.0214). This might be because some parts of the images in the genuine pair were contaminated resulting in spurious and missing minutiae, and the images in the imposter pair have similar pattern type. On the other hand, the pore matcher found an obviously larger number of mated pores on the genuine pair than on the imposter pair (see Fig. 10(b) and Fig. 10(d)). After fusion of pores and VeriFinger, the match score of the genuine pair is improved to 0.0571 , whereas that of the imposter pair is just 0.0396 . Note that there are still some falsely mated pores found by the pore matcher, and the recognition accuracy is thus expected to be further enhanced by improving the pore extractor and matcher.

\section{Conclusions}

We have studied the utility of pores, one of the most prevalent extended fingerprint features, on rolled ink fingerprint images. Pores are believed to be routinely used by forensic experts for latent matching. Although most previous studies claimed that pores are effective in improving the accuracy of minutiae matcher, they have following limitations: (i) used high resolution (>1000ppi) live-scan fingerprint images whose quality is generally very good and intra-class variation is small compared to rolled inked impressions; (ii) did not use a commercial state-of-the-art minutiae matcher as a baseline. This study is based on rolled ink fingerprint images which are widely used in forensic applications. Fingerprint images of three different quality at two different resolutions (500ppi and 1000ppi) were considered in our experiments. By using NIST SD30 database, a commercial minutiae matcher (VeriFinger), and state-of-the-art pore extraction and matching algorithms, we investigated the impact of fingerprint image quality on the accuracy of automatic pore extraction, and the effectiveness of pores in improving fingerprint recognition accuracy. Our experimental results show that the (i) automatic pore extraction and matching accuracy is significantly affected by fingerprint image quality, (ii) pores do not provide any significant improvement to the fingerprint recognition 
accuracy on 500ppi fingerprint images, and (iii) fusion of pore and minutiae matchers is effective only for high resolution (1000ppi) fingerprint images of good quality. In general, it is recommended that pores be used only for high resolution fingerprint images of good quality. As observed in this study, even COTS minutiae matchers are

(a)

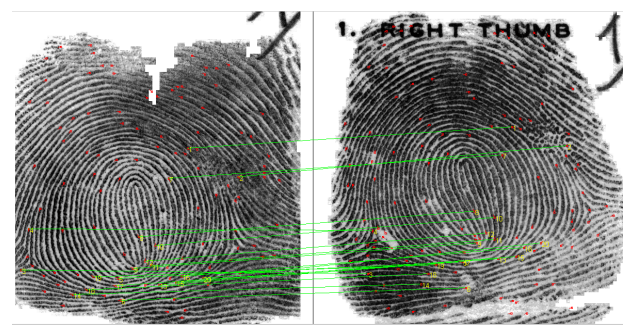

(c)

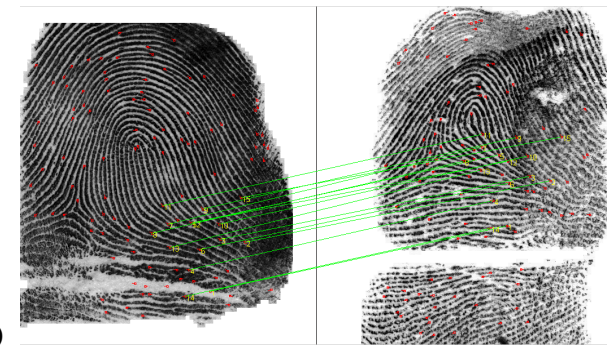

not perfect in recognizing good quality fingerprints. In our ongoing study, we are attempting to improve the performance of automatic pore extraction and matching and investigating better fusion strategy for combining pore and minutiae matchers. We also plan to examine the utility of ridge edges, another prevalent extended feature.

(b)

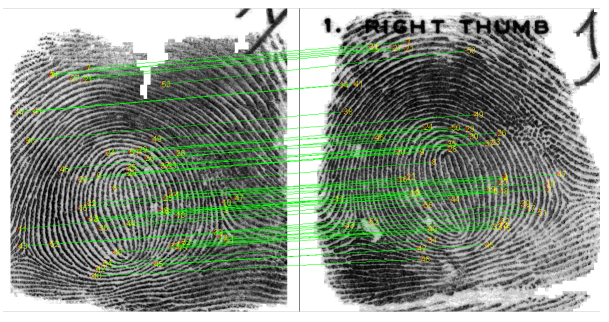

(d)

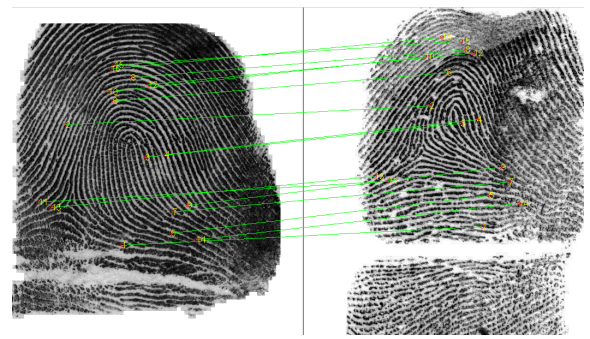

Fig. 10: (a) and (b) show a genuine pair with mated minutiae found by VeriFinger and mated pores found by the pore matcher. (c) and (d) show an imposter pair with mated minutiae found by VeriFinger and mated pores found by the pore matcher.

\section{References}

[1] H. C. Lee and R. E. Gaensslen (eds.). Advances in Fingerprint Technology, $2^{\text {nd }}$ Edition, Elsevier, 2001.

[2] D. Maltoni, D. Maio, A. K. Jain, and S. Prabhakar. Handbook of Fingerprint Recognition, $2^{\text {nd }}$ Edition, Springer-Verlag, 2009.

[3] D. R. Ashbaugh. Quantitative-Qualitative Friction Ridge Analysis: An Introduction to Basic and Advanced Ridgeology, CRC Press, 1999.

[4] A. Roddy and J. Stosz. Fingerprint features - Statistical analysis and system performance estimates. Proceedings of the IEEE, 85 (9): 1390-1421, 1997.

[5] SWGFAST. http://fingerprint.nist.gov/standard/cdeffs/Docs/SWGFAST _Memo.pdf, 2005.

[6] FBI. Electronic Fingerprint Transmission Specification (EFTS), Version 7.1, May, 2005 http://www.fbi.gov/hq/cjisd/iafis/efts71/cover.htm.

[7] CDEFFS. Data Format for the Interchange of Extended Fingerprint and Palmprint Features, Draft Version 0.4, June 2009, http://fingerprint.nist.gov/standard/cdeffs/index.html.

[8] J. D. Stosz and L. A. Alyea. Automated system for fingerprint authentication using pores and ridge structure. Proc. of SPIE, San Diego, vol. 2277, pp. 210-223, 1994.

[9] K. Kryszczuk, P. Morier, and A. Drygajlo. Study of the distinctiveness of level 2 and level 3 features in fragmentary fingerprint comparison. Proc. of Biometric Authentication Workshop, ECCV 2004, pp. 124-133, 2004.
[10] A. K. Jain, Y. Chen, and M. Demirkus. Pores and ridges: Fingerprint matching using level 3 features. IEEE Trans. PAMI, 29(1): 15-27, 2007.

[11] Q. Zhao, L. Zhang, D. Zhang, and N. Luo. Adaptive fingerprint pore modeling and extraction. Pattern Recognition, to appear, 2010.

[12] Q. Zhao, L. Zhang, D. Zhang, and N. Luo. Direct pore matching for fingerprint recognition. Proc. of ICB, Italy, pp. 597-606, 2009.

[13] A. K. Jain and J. Feng. Latent fingerprint matching. IEEE Trans. PAMI, to appear, 2010

[14] E. Tabassi, C.Wilson, and C.Watson. Fingerprint image quality. NIST Res. Rep. NISTIR7151, 2004.

[15] H. Fronthaler, K. Kollreider, J. Bigun, J. Fierrez, F. AlonsoFernandez, J. Ortega-Garcia, J.Gonzalez-Rodriguez. Fingerprint image-quality estimation and its application to multialgorithm verification. IEEE Trans. IFS, 3(2): 331-338, 2008.

[16] Neurotechnology. VeriFinger $\quad$ SDK. http://www.neurotechnology.com/verifinger.html.

[17] A. Ross, K. Nandakumar, and A. K. Jain. Handbook of Multibiometrics, Springer, 2006.

[18] K. Nandakumar, Y. Chen, S. C. Dass, and A. Jain. Likelihood ratio-based biometric score fusion. IEEE Trans. PAMI, 30(2): 342-347, 2008. 